Published in final edited form as:

Atherosclerosis. 2020 January ; 293: 1-10. doi:10.1016/j.atherosclerosis.2019.11.034.

\title{
Genetic linkage of oxidative stress with cardiometabolic traits in an intercross derived from hyperlipidemic mouse strains
}

\author{
Daniela T. Fullera ${ }^{\mathrm{a}}$, Andrew T. Grainger ${ }^{\mathrm{b}}$, Ani Manichaikul ${ }^{\mathrm{b}, \mathrm{c}}$, Weibin Shi ${ }^{\mathrm{a}, \mathrm{b}, 1}$ \\ aDepartments of Radiology \& Medical Imaging, University of Virginia, Charlottesville, Virginia, \\ USA. \\ bBiochemistry \& Molecular Genetics, University of Virginia, Charlottesville, Virginia, USA. \\ ${ }^{\circ}$ Center for Public Health Genomics, University of Virginia, Charlottesville, Virginia, USA.
}

\begin{abstract}
Background and aims: Oxidative stress is associated with cardiometabolic traits in observational studies, yet the underlying causal relationship remains unclear. Apolipoprotein Edeficient $\left(\mathrm{Apoe}^{-/-}\right)$mice develop significant hyperlipidemia and hyperglycemia on a Western diet. Here we conducted linkage analysis to investigate genetic connections between cardiometabolic traits and oxidative stress.
\end{abstract}

Methods: 266 female F2 mice were generated from an intercross between C57BL/6 (B6) and BALB/c (BALB) Apoe ${ }^{-/-}$mice and fed 12 weeks of Western diet. Plasma levels of HDL, LDL cholesterol, triglycerides, glucose and malondialdehyde (MDA) and atherosclerosis in aortic root and left carotid artery were measured. 127 microsatellite markers across the genome were genotyped.

Results: One significant locus at $78.3 \mathrm{cM}$ on chromosome (Chr) 1 (LOD score: 3.85), named Mda1, and two suggestive loci near $60.3 \mathrm{cM}$ on Chr1 (LOD score: 2.32, namedMda2 due to replication in a separate cross) and $19.6 \mathrm{cM}$ on Chr4 (LOD score: 2.34) were identified for MDA levels. Mda1 coincided precisely with loci for LDL, triglyceride, glucose, and body weight and overlapped with a locus for atherosclerosis in the aortic root. Plasma LDL, triglyceride, and glucose explained 25.5, 19.2, and 24.2\% of the variation in MDA levels of F2 mice, respectively. After correction for triglyceride or LDL, QTLs for MDA on Chr1 and Chr4 disappeared. QTLs on

\footnotetext{
${ }^{1}$ Correspondence to: Weibin Shi, MD, PhD, University of Virginia, Snyder Bldg Rm 266, 480 Ray C. Hunt Dr, P.O. Box 801339, Charlottesville, VA 22908, Fax: (434) 924 9435, Phone: (434) 243 9420, ws4v@ virginia.edu.

Author contributions: Daniela Fuller, Andrew Grainger, Weibin Shi conceived the study, designed and performed the experiments, analyzed the data, and drafted the manuscript. Ani Manichaikul supervised data analysis.

Conflicts of interest: The authors declared they do not have anything to disclose regarding conflict of interest with respect to this manuscript.

Conflict of interest

Authors declared no conflict of interest, including financial, personal or other relationships with other people or organizations, within the past three years that could have inappropriately influenced the work reported here.

Supplementary data: All data used in this article can be found in Supplementary materials.

Publisher's Disclaimer: This is a PDF file of an unedited manuscript that has been accepted for publication. As a service to our customers we are providing this early version of the manuscript. The manuscript will undergo copyediting, typesetting, and review of the resulting proof before it is published in its final form. Please note that during the production process errors may be discovered which could affect the content, and all legal disclaimers that apply to the journal pertain.
} 
Chr1 disappeared, remained on Chr4, and additional QTLs on Chr12 and Chr13 were detected after correction for glucose. The QTL on Chr12, named Mda3, had a significant LOD score of 8.034 and peaked 62.22 at $\mathrm{cM}$.

Conclusions: We demonstrated a causative role for cardiometabolic traits in oxidative stress and identified hyperlipidemia and hyperglycemia as a major driver of oxidative stress.

\section{Graphical Abstract}

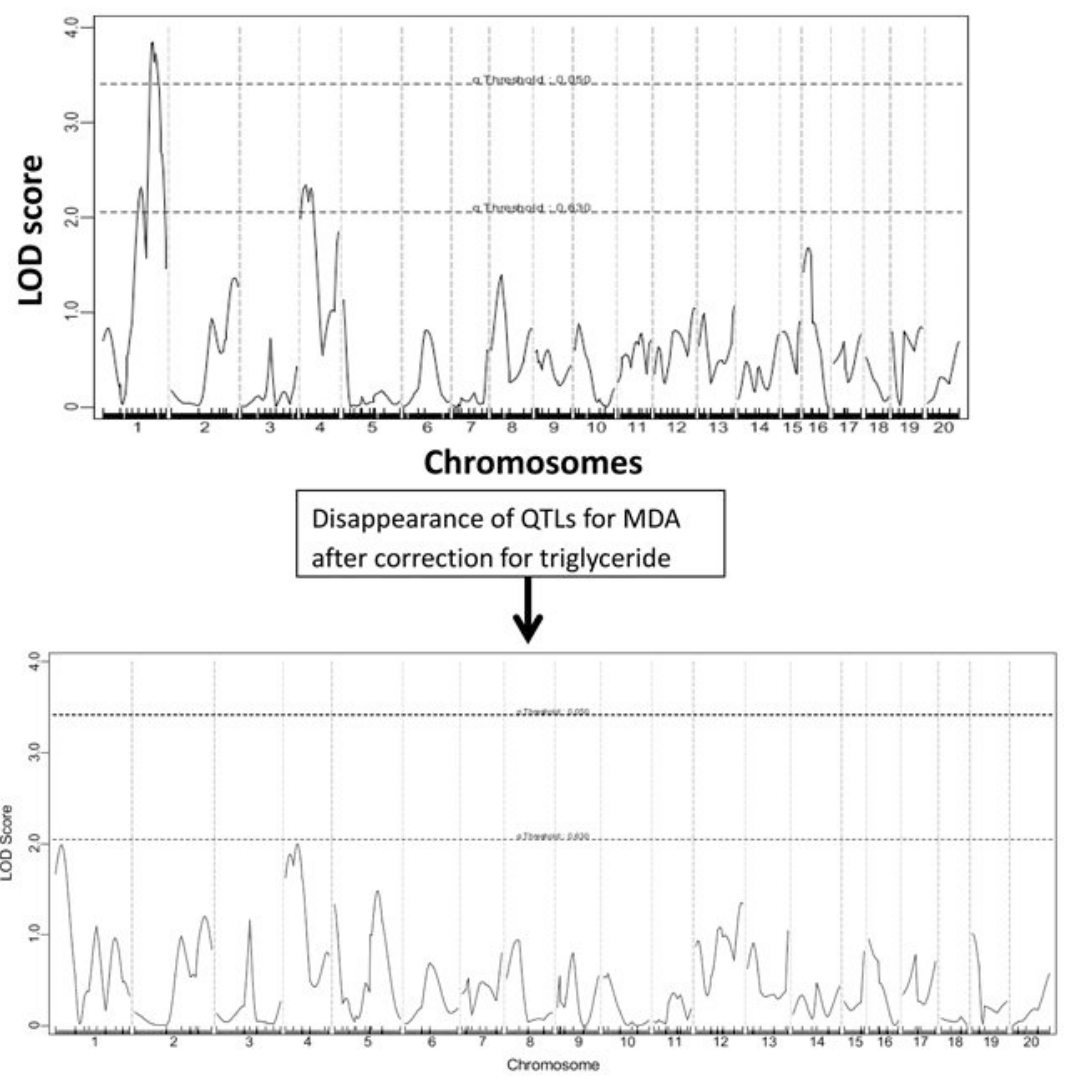

\section{Introduction}

Cardiometabolic disorders represent a cluster of interrelated risk factors, primarily dyslipidemia, hyperglycemia, abdominal obesity and hypertension that predispose to both cardiovascular diseases and diabetes, and affect $35 \%$ of US adults [1]. Dyslipidemia, including elevations in triglyceride and LDL cholesterol levels and reductions in HDL cholesterol levels, plays a major role in the development of atherosclerosis. Large scale population studies have associated different plasma lipid components with the risk for cardiovascular events, with most studies focusing on total, LDL, HDL cholesterol and triglyceride [2],[3]. Genome-wide association studies (GWAS) have identified over 60 common genetic variants significantly associated with coronary artery disease, and a large fraction of these loci harbor the genes that affect LDL, HDL cholesterol and triglyceride levels [4]. Lipid-lowering statin and anti-PCSK9 antibody therapies have provided definitive proof for a causal role of hyperlipidemia in atherosclerosis [5],[6]. Dyslipidemia is also 
associated with increased risk for other common non-communicable diseases, such as type 2 diabetes [7],[8] and Alzheimer's disease [9].

Oxidative stress resulting from increased production of reactive oxygen/nitrogen species (ROS) and/or reduced antioxidant capacity is considered an important mechanism underlying the pathogenesis of many common non-communicable diseases, including atherosclerosis, type 2 diabetes, neurodegenerative diseases, and cancer [10]. ROS are reactive intermediates of oxygen produced from various metabolic processes in the cells under physiological conditions and involved in such essential biological processes as stressor response and inflammation [11]. Free superoxide radical $\left(\mathrm{O}_{2}{ }^{--}\right)$is the primary species produced from the mitochondrial respiratory chain in the cells and is the source of other reactive species of pathological significance such as $\mathrm{H}_{2} \mathrm{O}_{2}, \mathrm{OH} \bullet$, and $\mathrm{ONOO}^{-}$. When an aberrant production of ROS exceeds the buffering capacity of the antioxidant defense systems, oxidative stress occurs, leading to oxidative damages to proteins, nucleotides, and lipids. Observational studies have shown that alterations in lipid metabolism associate with redox imbalance [12],[13],[14],[15],[16]. Individuals with hyperlipidemia, higher triglyceride or lower HDL cholesterol levels have a pro-oxidant status [12],[13],[14], [15], [16],[17]. Hyperlipidemia patients receiving aggressive lipid lowering therapy have decreased lipoperoxidation [18]. However, no studies have been conducted to define the genetic basis underlying the associations between oxidative stress and cardiometabolic traits. Therefore, we performed linkage analysis to investigate whether there are genetic connections between oxidative stress and cardiometabolic traits using a segregating $\mathrm{F} 2$ population derived from C57BL/6 (B6) and BALB/cJ (BALB) Apoe ${ }^{-/-}$mice.

\section{Materials and methods}

\section{Mice}

The generation of a female F2 cohort from an intercross between B6- $A p o e^{-/}$and BALB$A$ poe $^{-/-}$mice was performed as reported [19]. F2 mice were weaned at 3 weeks of age onto a rodent chow diet and switched onto a Western diet containing $21 \%$ fat, $34.1 \%$ sucrose, $0.15 \%$ cholesterol, and $19.5 \%$ casein (Evigo, TD 88137) at 6 weeks of age. After 12 weeks on the Western diet, blood was collected from overnight-fasted mice under isoflurane anesthesia. All procedures were performed in accordance with current National Institutes of Health guidelines and approved by the Institutional Animal Care and Use Committees.

\section{Measurements of plasma malondialdehyde (MDA), lipids, glucose, and atherosclerosis}

Plasma MDA was measured with a Cayman TBARS (Thiobarbituric Acid Reactive Substances) kit (Cat. \# 10009055). Plasma glucose was determined with a Sigma assay kit (Cat. \# GAHK20). Total and HDL cholesterol and triglyceride concentrations were measured enzymatically using Thermo DMA (Louisville, CO) cholesterol and triglyceride kits. Non-HDL cholesterol concentrations were calculated as the difference between total and HDL cholesterol levels. Atherosclerotic lesion sizes in the aortic root and left carotid arteries of mice were measured as reported [19],[20]. 


\section{Genotyping}

Genomic DNA was isolated from tail clips of mice and genotyped as described [19]. A total of 127 microsatellite markers across the entire genome at an average interval of $12 \mathrm{cM}$ were typed.

\section{Statistical analysis}

QTL analysis was performed using the standard analysis software J/qtl and Map Manager QTX as we previously reported [19],[21],[22]. One thousand permutations of trait values were run to define the genome-wide LOD (logarithm of odds) score threshold for significant or suggestive linkage of a particular trait. Loci that exceeded the LOD score threshold of 0.05 were considered significant $(p<0.05)$ and those exceeding the threshold of 0.63 were suggestive $(p<0.63)$. To evaluate a potential causal relationship between overlapping QTLs for two different traits, the residuals derived from linear regression analysis of the two traits were used as a new phenotype. The same mapping algorithm used previously was then performed for the new phenotype. Alternatively, the metabolic trait or genetic marker of interest was used as an interactive covariate when performing the mapping algorithms so that its effect on another trait was eliminated. These two strategies help define QTL independence from another trait or even expose a masked QTL. Regression analysis was performed to dissect the contribution of an individual metabolic trait to the variation in plasma MDA levels of F2 mice. Heritability for plasma MDA was determined through marker regression analysis by summing up percent phenotypic variance explained by each QTL.

\section{Results}

\section{Genetic control and heritability of plasma MDA levels}

A genome-wide scan was performed to search for QTLs for plasma MDA levels of the F2 mice. One significant QTL on chromosome (Chr) 1 and two suggestive QTLs on Chr1 and Chr4 were identified for MDA (Fig. 1, Table 1). Details of these QTLs, including locus name, LOD score, 95\% confidence interval (CI), peak location, genome-wide significance probability value, high allele, and mode of inheritance are presented in Table 1. The significant QTL on Chr1 had a significant LOD score of 3.85 and peaked at $78.31 \mathrm{cM}$. We named itMda1 to represent the first significant QTL mapped for mouse plasma MDA. This locus exhibited an additive inheritance pattern in that F2 mice homozygous for the BALB (CC) allele at peak marker D1Mit354 had higher plasma MDA levels than those homozygous for the B6 (BB) allele while F2 mice heterozygous for the allele had an intermediate MDA level (Table 1).

The suggestive QTL on Chr1 had an LOD score of 2.32 and peaked at $60.31 \mathrm{cM}$ (Table 1). As this QTL was replicated in a BALB x SM/J intercross (unpublished data), we named it Mda2 according to guidelines on the Standardized Genetic Nomenclature for rodents. This QTL affected plasma MDA levels in a dominant manner of inheritance from the BALB allele (Table 1). The Chr4 locus had a suggestive LOD score of 2.34 and peaked at 19.55 cM. This QTL affected plasma MDA levels in an additive mode with the B6 allele elevating and BALB allele lowering MDA levels (Table 1). 
The heritability of plasma MDA levels was obtained for the F2 cross using the marker regression analysis by summing up the percent phenotypic variance explained by each QTL. The QTLs with an LOD score $\geq 1.35$ accounted for $37 \%$ of the variation in plasma MDA levels of the cross (Supplementary materials).

\section{Colocalization of QTLs for plasma MDA with QTLs for LDL, triglyceride, glucose, body weight, and atherosclerosis}

LOD score plots for Chr1 show that the two QTLs for plasma MDA (Mda1, Mda2) coincided precisely with QTLs for plasma LDL cholesterol levels in the confidence interval (Fig. 2). The distal QTL for LDL overlapped with $C q 1$, a locus originally identified in a B6 $\times$ KK-Ay intercross for plasma cholesterol concentrations [23]. We previously named the proximal QTL for LDL Nhdlq13 to represent a significant QTL for non-HDL cholesterol in the mouse [20]. For both QTLs, the BALB allele was associated with elevated and the B6 allele with decreased LDL cholesterol levels.

The QTL for triglyceride, $T g l q 1$, peaked at $74.31 \mathrm{cM}$ and overlapped with $M d a 1$ in the confidence interval (Fig. 2). The QTL for plasma glucose on distal Chr1 replicates Bglu3, originally mapped in a $\mathrm{B} 6 \times \mathrm{C} 3 \mathrm{H} A p o e^{-/-}$intercross [24]. The BALB allele was associated with elevated and the B6 allele with decreased triglyceride and glucose levels.

The distal QTL Mda1 also overlapped with a QTL for body weight and Ath1, a QTL affecting aortic atherosclerotic lesion size, in the confidence interval (Fig. 2). The BALB allele was associated with a heavier body weight and a smaller lesion size and the B6 allele with a lighter body weight and a larger lesion size (Fig. 2). Bw8q1, a locus for body weight, was originally mapped to this region in a B6 $\times \mathrm{A} / \mathrm{J}$ intercross [25] and then replicated in a B6 x C3H Apoe ${ }^{-/-} \mathrm{F} 2$ cross [24]. Wt3q2 and Wt6q2 for body weight have also been mapped to this region in two F2 populations derived from mouse lines differing in heat loss [26]. Ath1, a locus that affects atherosclerotic lesion size and HDL cholesterol levels, was initially mapped in recombinant inbred strains derived from B6 and BALB and also from B6 and $\mathrm{C} 3 \mathrm{H} / \mathrm{HeJ}$ [27] and replicated in several intercrosses derived from $A p o e^{-/}$mouse strains [28] [29][30].

\section{Dissection of the contribution of cardiometabolic traits to variation in plasma MDA levels}

The coefficient of determination, $R^{2}$, a key output of regression analysis, estimates the proportion of the variance in one variable that is accounted for by the other [31]. The potential contribution of plasma LDL, HDL cholesterol, triglyceride, glucose, and body weight to variation in plasma MDA levels was evaluated using the F2 population (Fig. 3). Based on the $R^{2}$ values, LDL, triglyceride, and glucose explained $25.5 \%, 19.2 \%$, and $24.2 \%$ of the variance in plasma MDA levels among the F2 mice, respectively. Plasma HDL cholesterol, body weight, and aortic atherosclerotic lesion sizes had no significant associations with plasma MDA levels in the F2 mice $(p>0.05)$. In contrast, a slight but statistically significant association was observed between plasma MDA levels and carotid atherosclerotic lesion sizes in the F2 mice $\left(p=0.0073 ; R^{2}=0.029\right)$. F2 mice with higher MDA levels had larger carotid lesion sizes than those with lower MDA levels. Based on the $R^{2}$ value, plasma MDA accounted for $2.9 \%$ of the variance in carotid lesion size of F2 mice. 


\section{Dissection of the causal relationship between metabolic traits and plasma MDA levels}

As aforementioned, plasma MDA levels were significantly correlated with plasma levels of triglyceride, LDL cholesterol, and glucose with each explaining $\sim 20 \%$ of the variation. To determine whether variation in MDA levels was dependent on one or more of the metabolic traits, we generated the residuals from the linear regression of MDA levels with each of the 3 traits and used the residuals as a new phenotype for QTL mapping. When the residuals from the regression analysis with triglyceride were analyzed, no QTL for MDA levels was found (Fig.4A), implying the dependence of MDA QTLs on triglyceride. When residuals from the regression with LDL were analyzed, the QTLs on Chr1 and Chr4 for MDA shown above in Fig. 1 disappeared, suggesting the dependence of these QTLs on LDL (Fig. 4B). Besides, two suggestive QTLs on Chr12 emerged, which peaked at 38.59 and $62.22 \mathrm{cM}$ (Fig. 4B, Table 1). When residuals from the regression with glucose levels were analyzed, the QTLs for MDA on Chr1 disappeared, QTL on Chr4 remained, and the 2 suggestive QTLs on Chr12 also emerged (Fig. 4C), implying the dependence of Chr1 QTLs on glucose and the independence of $\mathrm{Chr} 4$ and Chr12 QTLs on glucose.

The same mapping algorithms used above in Fig. 1 were also performed except for the use of one metabolic trait or a genetic marker as an interactive covariate (Fig. 4D 4G). When triglyceride was used as a covariate, no QTL was detected for MDA levels (Fig. 4D). When LDL was a covariate, QTLs on Chr1 and Chr4 for MDA disappeared and a QTL on distal Chr12 was detected (Fig. 4E). The Chr12 QTL, namedMad3, had a significant LOD score of 4.78 and peaked at $62.22 \mathrm{cM}$. When glucose levels were used as a covariate, the QTLs on Chr1 disappeared, the QTL on Chr4 remained, and QTLs on Chr12 (Mda3) and Chr13 were detected (Fig. 4F). The Chr12 QTL, Mad3, had a significantly higher LOD score of 8.03 (Table 1). The Chr13 QTL had a suggestive LOD score of 4.28 and peaked at $21.99 \mathrm{cM}$.

The same QTL mapping algorithm used for Fig. 1 was also performed with D1Mit345, the peak genetic marker for Mda1 and also for QTLs for triglyceride, LDL and glucose, as an interactive covariate. As shown in Fig. 4G, all 3 QTLs for MDA remained but their LOD scores were diminished compared to those shown in Fig. 1.

\section{Discussion}

Dyslipidemia and hyperglycemia are integral components of the metabolic perturbations in type 2 diabetes, obesity, and the metabolic syndrome and constitute major risk factors for cardiovascular disease. Our present study identified multiple QTLs for plasma levels of malondialdehyde (MDA), a circulating biomarker of oxidative stress, through the use of a segregating F2 population derived from two hyperlipidemic $A p o e^{-/}$mouse strains. We observed the colocalization of QTLs for MDA levels with QTLs for plasma levels of LDL cholesterol, triglyceride, and glucose, body weight, and atherosclerosis on chromosome 1. Moreover, plasma MDA levels were found positively correlated with LDL cholesterol, triglyceride, and glucose levels, to a large extent, and with carotid atherosclerosis, but were not with HDL cholesterol levels, aortic atherosclerotic lesion sizes or body weight in the F2 population. Interestingly, the QTLs for MDA on chromosome 1 disappeared after correction for plasma triglyceride, LDL or glucose levels. 
B6 and BALB $\mathrm{Apoe}^{-/-}$mice exhibit distinct differences in atherosclerosis, LDL, HDL cholesterol, and type 2 diabetes-related traits [32],[33]. In a female F2 cohort derived from the two $\mathrm{Apoe}^{-/-}$strains, we mapped dozens of loci for atherosclerosis, body weight, plasma lipids and glucose [19],[20]. We have now demonstrated that genetic factors determine the magnitude of oxidative stress for the first time in animals. Although oxidative stress is associated with the development of many common chronic diseases, including atherosclerosis and type 2 diabetes, it remains unknown whether there is a genetic basis in the process. Interval mapping plots for chromosome 1 showed two distinct peaks of the linkage curve for plasma MDA levels with the distal peak appearing at $78.3 \mathrm{cM}$ and the proximal peak at $60.3 \mathrm{cM}$. The bootstrap test, a statistical method for estimating the confidence interval of QTLs using simulation [34], supports the existence of two QTLs for plasma MDA on chromosome 1. We named the distal QTL Mda1 to represent the first significant locus for plasma MDA in the mouse. Although the proximal QTL had a suggestive LOD score, it has been replicated in a separate cross and thus named Mda2. Mda1 and Mda2 coincided precisely with QTLs for LDL cholesterol in the confidence interval. Mda1 is also overlapping with QTLs for triglyceride, glucose, body weight, and aortic atherosclerosis. The distal QTL for LDL replicates $C q 1$, initially mapped in a B6 x KK-Ay/a F2 cohort [23], and the proximal QTL for LDL replicates Nhdlq13 we previously mapped in a B x H intercross [20]. The co-localization of QTLs for two different traits suggests a possibility that the traits be controlled by the same gene with pleiotropic effects [35]. The finding that both QTLs exhibited a higher effect from the BALB allele on LDL and MDA supports this speculation. Indeed, F2 mice with the BALB allele at the locus had both higher LDL and MDA levels than those with the B6 allele. We and others have identified Apoa2 and Soat 1 as the causal gene of $C q 1$ and Nhdlq13, respectively [36],[37], [38][39]. Indeed, Apoa2 is a major gene affecting plasma lipid levels in the mouse [37]. APOA2 polymorphism has shown associations with plasma markers of oxidative stress in type 2 diabetic patients [40]. The QTL for plasma glucose replicates Bglu3, initially mapped in a $\mathrm{B}$ x H cross [24]. Apcs, encoding serum amyloid P-component (SAP), and Apoa2 are the likely causal genes of Bglu3 [38],[41],[42]. SAP is an acute phase response plasma protein in the mouse and is equivalent to C-reactive protein (CRP) in humans. Oxidative stress markers were found significantly correlated with blood glucose in prediabetic human subjects [43].

Strong correlations of plasma MDA levels with LDL cholesterol and triglyceride levels were found in the F2 cross. The $R^{2}$ value achieved from regression analysis represents the contribution of a variable to the variation of another. Based on $R^{2}$ values, LDL accounted for $25.5 \%$ and triglyceride for $19.2 \%$ of the variation in plasma MDA levels of the F2 population. Accordingly, hyperlipidemic patients had an increased MDA level compared to normolipidemic individuals and normolipidemic subjects with higher triglycerides, total and LDL cholesterol levels also had higher MDA levels than those who had lower lipid levels [44]. In cultured human cells, native LDL caused a marked increase of ROS production by the mitochondria and activation of the NADPH oxidase [45].

A significant positive correlation was observed between plasma MDA levels and fasting glucose levels in this cross. The strong correlation between the traits has also been observed in prediabetic human subjects [46]. Elevated blood glucose activates a metabolic route that 
involves diacylglycerol, protein kinase $\mathrm{C}$, and NADPH-oxidase, leading to culmination of ROS [47]. The latter causes oxidative damage to DNA, lipids, and proteins. Lipid peroxidation forms relatively stable end products such as MDA and isoprostanes [48].

Although Mda1 overlaps with QTLs for body weight (BW8q1), HDL (Hdlq6), and aortic atherosclerosis $(A t h I)$ in the confidence interval on distal chromosome 1, plasma MDA shows no correlation with body weight, HDL cholesterol levels, or aortic lesion sizes in the F2 cohort. A small but statistical correlation between MDA levels and carotid atherosclerotic lesion sizes was observed in this cross, but no QTL for carotid atherosclerosis was found in distal chromosome 1 region [20]. A likely explanation for the poor or lack of MDA correlation with aortic or carotid atherosclerotic lesions is absent of meaningful genetic connections between the traits. Indeed, analysis of marker regression data shows that there were no or few shared marker loci throughout the entire genome that affected both traits (Supplementary data). In contrast, for the traits LDL, triglyceride, and glucose that showed strong correlations with plasma MDA, there were shared marker loci that affected the correlated traits. Thus, the QTL and association data strongly suggest that LDL, triglyceride, and glucose play a causal role in oxidative stress during dyslipidemia.

Two strategies have been utilized to define the causal relationship between plasma MDA levels and metabolic traits, i.e. 1) creating residuals from the linear regression of MDA levels with one of the metabolic traits, and then mapping QTLs on the residuals so as to find genetic factors accounting for variation in MDA other than that metabolic trait, and 2) using one of the metabolic traits as an interactive covariate during QTL scanning so that its effect on MDA levels were excluded. With these two methods, we demonstrated that the MDA QTLs on Chr1 and Chr4 were triglyceride- and LDL-dependent, the Chr1 QTLs were also glucose-dependent while QTLs on Chr4, Chr12 and Chr13 were glucose-independent. The use of the second method has shown an additional power in revealing QTLs on Chr12 and Chr13 that would have not been found with the traditional methods.

A significant QTL, Mda3, for MDA was discovered after adjustment for plasma LDL or glucose levels. Because a QTL arises from changes in the function or the quantity of a gene product, we searched for genes that carry one or more nonsynonymous coding SNPs or a SNP in the upstream regulatory region within the confidence interval of Mda3. Notably, there is a gene cluster that consists of $>100$ genes encoding for the immunoglobulin heavy chain (IGH). These genes are highly polymorphic, with each containing one or more nonsynonymous SNPs or a SNP in the upstream regulatory region segregating between the B6 and BALB parental strains (supplementary data). Consistently, a recent study shows that B6 and BALB mice share very few IGH variable sequences [52]. High fat diet feeding, which induces oxidative stress, has been shown to markedly decrease the number of B cells in the blood and spleen and suppress the expression of IGH-6 and IGH $\gamma$-polypeptide in the spleen of mice [53]. Together, these data suggest a possibility that IGH is involved in regulating oxidative stress.

Plasma MDA only marginally associated with carotid atherosclerotic lesion sizes (accounted for $2.9 \%$ of the variation) and showed no association with aortic atherosclerosis in the cross. Similarly, LDL, triglyceride, and glucose, the established risk factors for atherosclerosis, are 
also poorly associated with carotid or aortic atherosclerotic lesion sizes in this cross (supplementary data) and other crosses derived from $A p o e^{-/-}$mouse strains [49][30][21][50] [20][51]. One likely explanation for poor or absent of correlation between atherosclerosis and its major risk is that both of them are polygenetic traits and controlled by multiple genes while most of the genetic loci are distinct for the traits. Indeed, in this cross carotid atherosclerosis is controlled by QTLs on Chr5, Chr12, Chr13, Chr17, and Chr18 [20] and thus these QTLs are different from those for MDA. Aortic atherosclerosis is controlled by significant QTLs on Chr1, Chr2, Chr5, and Chr9 [19], and these QTLs are also different from those MDA QTLs except for the Chr1 locus. Similarly, in crosses in which atherosclerotic lesion sizes are poorly associated plasma lipids, there is a lack or rarity of overlapping QTLs between the two traits [49][30][21][50][20][51].

Short-lived ROS are difficult to measure directly as they quickly react with endogenous antioxidants, proteins, nucleotides, and lipids. The oxidation of polyunsaturated fatty acids in lipids leads to the formation of MDA, which can be accurately measured by the TBARS assay. In this study, we found that MAD levels were strongly correlated with plasma triglyceride, LDL, and glucose levels in the cross. Indeed, F2 mice with higher triglyceride, LDL or glucose levels had higher MDA levels than those that had lower levels of the traits. The mapping data demonstrated the essentiality of the metabolic traits to the production of MDA in hyperlipidemic mice, since the correction of either trait led to the disappearance of major QTLs for MDA.

In summary, we identified plasma levels of MDA as a polygenic trait controlled by multiple QTLs, demonstrated a causative role for cardiometabolic traits in oxidative stress, and identified hyperlipidemia and hyperglycemia as a major driver of oxidative stress in a segregating F2 population.

\section{Acknowledgments:}

This work was supported by NIH grants R01 DK116768 and HL112281 and Commonwealth Health Research Board (CHRB) Virginia.

\section{References}

[1]. Alberti KG, Eckel RH, Grundy SM, Zimmet PZ, Cleeman JI, Donato KA, et al., Harmonizing the metabolic syndrome: a joint interim statement of the International Diabetes Federation Task Force on Epidemiology and Prevention; National Heart, Lung, and Blood Institute; American Heart Association; World Heart Federation; International Atherosclerosis Society; and International Association for the Study of Obesity, Circulation 120(2009) pp. 1640-5. [PubMed: 19805654]

[2]. Sharrett AR, Ballantyne CM, Coady SA, Heiss G, Sorlie PD, Catellier D, et al., Coronary heart disease prediction from lipoprotein cholesterol levels, triglycerides, lipoprotein(a), apolipoproteins A-I and B, and HDL density subfractions: The Atherosclerosis Risk in Communities (ARIC) Study, Circulation 104(2001) pp. 1108-13. [PubMed: 11535564]

[3]. Ridker PM, Rifai N, Cook NR, Bradwin G, Buring JE, Non-HDL cholesterol, apolipoproteins A-I and B100, standard lipid measures, lipid ratios, and CRP as risk factors for cardiovascular disease in women, JAMA294(2005) pp. 326-33. [PubMed: 16030277]

[4]. McPherson R, Tybjaerg-Hansen A, Genetics of Coronary Artery Disease, Circ Res 118(2016) pp. 564-78. [PubMed: 26892958] 
[5]. Pedersen TR, Kjekshus J, Berg K, Haghfelt T, Faergeman O, Faergeman G, et al., Randomised trial of cholesterol lowering in 4444 patients with coronary heart disease: the Scandinavian Simvastatin Survival Study (4S). 1994, Atheroscler Suppl 5(2004) pp. 81-7. [PubMed: 15531279]

[6]. Chaudhary R, Garg J, Shah N, Sumner A, PCSK9 inhibitors: A new era of lipid lowering therapy, World J Cardiol9(2017) pp. 76-91. [PubMed: 28289523]

[7]. Haffner SM, Miettinen H, Stern MP, Are risk factors for conversion to NIDDM similar in high and low risk populations? Diabetologia40(1997) pp. 62-6. [PubMed: 9028719]

[8]. Mykkanen L, Kuusisto J, Pyorala K, Laakso M, Cardiovascular disease risk factors as predictors of type 2 (non-insulin-dependent) diabetes mellitus in elderly subjects, Diabetologia36(1993) pp. 553-9. [PubMed: 8335178]

[9]. Reitz C, Dyslipidemia and the risk of Alzheimer's disease, Curr Atheroscler Rep15(2013) pp. 307,012-0307-3. [PubMed: 23328907]

[10]. Incalza MA, D'Oria R, Natalicchio A, Perrini S, Laviola L, Giorgino F, Oxidative stress and reactive oxygen species in endothelial dysfunction associated with cardiovascular and metabolic diseases, Vascul Pharmacol100(2018) pp. 1-19. [PubMed: 28579545]

[11]. Zuo L, Zhou T, Pannell BK, Ziegler AC, Best TM, Biological and physiological role of reactive oxygen species--the good, the bad and the ugly, Acta Physiol (Oxf)214(2015) pp. 329-48. [PubMed: 25912260]

[12]. Karabacak M, Varol E, Kahraman F, Ozaydin M, Turkdogan AK, Ersoy IH, Low high-density lipoprotein cholesterol is characterized by elevated oxidative stress, Angiology65(2014) pp. 92731. [PubMed: 24280265]

[13]. Rodriguez-Carrio J, Alperi-Lopez M, Lopez P, Lopez-Mejias R, Alonso-Castro S, Abal F, et al., High triglycerides and low high-density lipoprotein cholesterol lipid profile in rheumatoid arthritis: A potential link among inflammation, oxidative status, and dysfunctional high-density lipoprotein, J Clin Lipidol11(2017) pp. 1043,1054.e2. [PubMed: 28662934]

[14]. Swardfager W, Yu D, Scola G, Cogo-Moreira H, Chan P, Zou Y, et al., Peripheral lipid oxidative stress markers are related to vascular risk factors and subcortical small vessel disease, Neurobiol Aging59(2017) pp. 91-7. [PubMed: 28756941]

[15]. Fentoglu O, Kirzioglu FY, Bulut MT, Kumbul Doguc D, Kulac E, Onder C, et al., Evaluation of lipid peroxidation and oxidative DNA damage in patients with periodontitis and hyperlipidemia, J Periodontol86(2015) pp. 682-8. [PubMed: 25612631]

[16]. da Silva Pereira R, Tatsch E, Bochi GV, Kober H, Duarte T, dos Santos Montagner GF, et al., Assessment of oxidative, inflammatory, and fibrinolytic biomarkers and DNA strand breakage in hypercholesterolemia, Inflammation36(2013) pp. 869-77. [PubMed: 23436135]

[17]. Rahman T, Hamzan NS, Mokhsin A, Rahmat R, Ibrahim ZO, Razali R, et al., Enhanced status of inflammation and endothelial activation in subjects with familial hypercholesterolaemia and their related unaffected family members: a case control study, Lipids Health Dis16(2017) pp. 81,017-0470-1. [PubMed: 28438163]

[18]. Blaha V, Blaha M, Solichova D, Krcmova LK, Lanska M, Havel E, et al., Antioxidant defense system in familial hypercholesterolemia and the effects of lipoprotein apheresis, Atheroscler Suppl 30(2017) pp. 159-65. [PubMed: 29096832]

[19]. Zhang Z, Rowlan JS, Wang Q, Shi W, Genetic analysis of atherosclerosis and glucose homeostasis in an intercross between C57BL/6 and BALB/cJ apolipoprotein E-deficient mice, Circ Cardiovasc Genet5(2012) pp. 190-201. [PubMed: 22294616]

[20]. Rowlan JS, Zhang Z, Wang Q, Fang Y, Shi W, New quantitative trait loci for carotid atherosclerosis identified in an intercross derived from apolipoprotein E-deficient mouse strains, Physiol Genomics45(2013) pp. 332-42. [PubMed: 23463770]

[21]. Grainger AT, Jones MB, Li J, Chen MH, Manichaikul A, Shi W, Genetic analysis of atherosclerosis identifies a major susceptibility locus in the major histocompatibility complex of mice, Atherosclerosis254(2016) pp. 124-32. [PubMed: 27736672]

[22]. Garrett NE 3rd, Grainger AT, Li J, Chen MH, Shi W, Genetic analysis of a mouse cross implicates an anti-inflammatory gene in control of atherosclerosis susceptibility, Mamm Genome28(2017) pp. 90-9. [PubMed: 28116503] 
[23]. Suto J, Matsuura S, Yamanaka H, Sekikawa K, Quantitative trait loci that regulate plasma lipid concentration in hereditary obese KK and KK-Ay mice, Biochim Biophys Acta1453(1999) pp. 385-95. [PubMed: 10101257]

[24]. Su Z, Li Y, James JC, Matsumoto AH, Helm GA, Lusis AJ, et al., Genetic linkage of hyperglycemia, body weight and serum amyloid-P in an intercross between C57BL/6 and C3H apolipoprotein E-deficient mice, Hum Mol Genet15(2006) pp. 1650-8. [PubMed: 16595606]

[25]. Zhang S, Gershenfeld HK, Genetic contributions to body weight in mice: relationship of exploratory behavior to weight, Obes Res11(2003) pp. 828-38. [PubMed: 12855751]

[26]. Moody DE, Pomp D, Nielsen MK, Van Vleck LD, Identification of quantitative trait loci influencing traits related to energy balance in selection and inbred lines of mice, Genetics152(1999) pp. 699-711. [PubMed: 10353911]

[27]. Paigen B, Mitchell D, Reue K, Morrow A, Lusis AJ, LeBoeuf RC, Ath-1, a gene determining atherosclerosis susceptibility and high density lipoprotein levels in mice, Proc Natl Acad Sci U S A84(1987) pp. 3763-7. [PubMed: 3473481]

[28]. Zhang Z, Rowlan JS, Wang Q, Shi W, Genetic analysis of atherosclerosis and glucose homeostasis in an intercross between C57BL/6 and BALB/cJ apolipoprotein E-deficient mice, Circ Cardiovasc Genet5(2012) pp. 190-201. [PubMed: 22294616]

[29]. Wang SS, Schadt EE, Wang H, Wang X, Ingram-Drake L, Shi W, et al., Identification of pathways for atherosclerosis in mice: integration of quantitative trait locus analysis and global gene expression data, Circ Res101(2007) pp. e11-30. [PubMed: 17641228]

[30]. Rowlan JS, Li Q, Manichaikul A, Wang Q, Matsumoto AH, Shi W, Atherosclerosis susceptibility Loci identified in an extremely atherosclerosis-resistant mouse strain, J Am Heart Assoc2(2013) pp. e000260. [PubMed: 23938286]

[31]. Ludbrook J, Statistics in biomedical laboratory and clinical science: applications, issues and pitfalls, Med Princ Pract17(2008) pp. 1-13. [PubMed: 18059094]

[32]. Tian J, Pei H, James JC, Li Y, Matsumoto AH, Helm GA, et al., Circulating adhesion molecules in apoE-deficient mouse strains with different atherosclerosis susceptibility, Biochem Biophys Res Commun329(2005) pp. 1102-7. [PubMed: 15752767]

[33]. Liu S, Li J, Chen MH, Liu Z, Shi W, Variation in Type 2 Diabetes-Related Phenotypes among Apolipoprotein E-Deficient Mouse Strains, PLoS One10(2015) pp. e0120935. [PubMed: 25946019]

[34]. Visscher PM, Thompson R, Haley CS, Confidence intervals in QTL mapping by bootstrapping, Genetics143(1996) pp. 1013-20. [PubMed: 8725246]

[35]. Wagner GP, Kenney-Hunt JP, Pavlicev M, Peck JR, Waxman D, Cheverud JM, Pleiotropic scaling of gene effects and the 'cost of complexity', Nature452(2008) pp. 470-2. [PubMed: 18368117]

[36]. Lu Z, Yuan Z, Miyoshi T, Wang Q, Su Z, Chang CC, et al., Identification of Soat1 as a quantitative trait locus gene on mouse chromosome 1 contributing to hyperlipidemia, PLoS One6(2011) pp. e25344. [PubMed: 22022387]

[37]. Wang X, Korstanje R, Higgins D, Paigen B, Haplotype analysis in multiple crosses to identify a QTL gene, Genome Res14(2004) pp. 1767-72. [PubMed: 15310659]

[38]. Li J, Lu Z, Wang Q, Su Z, Bao Y, Shi W, Characterization of Bglu3, a mouse fasting glucose locus, and identification of Apcs as an underlying candidate gene, Physiol Genomics44(2012) pp. 345-51. [PubMed: 22274563]

[39]. Li J, Lu Z, Wang Q, Su Z, Bao Y, Shi W, Characterization of Bglu3, a mouse fasting glucose locus, and identification of Apcs as an underlying candidate gene, Physiol Genomics(2012).

[40]. Zamani E, Sadrzadeh-Yeganeh H, Sotoudeh G, Keramat L, Eshraghian M, Rafiee M, et al., The interaction between ApoA $2-265 \mathrm{~T}>\mathrm{C}$ polymorphism and dietary fatty acids intake on oxidative stress in patients with type 2 diabetes mellitus, Eur J Nutr56(2017) pp. 1931-8. [PubMed: 27271094]

[41]. Su Z, Li Y, James JC, Matsumoto AH, Helm GA, Lusis AJ, et al., Genetic linkage of hyperglycemia, body weight and serum amyloid-P in an intercross between C57BL/6 and $\mathrm{C} 3 \mathrm{H}$ apolipoprotein E-deficient mice, Hum Mol Genet15(2006) pp. 1650-8. [PubMed: 16595606] 
[42]. Castellani LW, Goto AM, Lusis AJ, Studies with apolipoprotein A-II transgenic mice indicate a role for HDLs in adiposity and insulin resistance, Diabetes50(2001) pp. 643-51. [PubMed: 11246886]

[43]. Mahat RK, Singh N, Rathore V, Arora M, Yadav T, Cross-sectional correlates of oxidative stress and inflammation with glucose intolerance in prediabetes, Diabetes Metab Syndr 13(2019) pp. 616-21. [PubMed: 30641776]

[44]. Yang RL, Shi YH, Hao G, Li W, Le GW, Increasing Oxidative Stress with Progressive Hyperlipidemia in Human: Relation between Malondialdehyde and Atherogenic Index, J Clin Biochem Nutr43(2008) pp. 154-8. [PubMed: 19015749]

[45]. Piccoli C, Quarato G, D'Aprile A, Montemurno E, Scrima R, Ripoli M, et al., Native LDLinduced oxidative stress in human proximal tubular cells: multiple players involved, J Cell Mol Med15(2011) pp. 375-95. [PubMed: 19863698]

[46]. Mahat RK, Singh N, Rathore V, Arora M, Yadav T, Cross-sectional correlates of oxidative stress and inflammation with glucose intolerance in prediabetes, Diabetes Metab Syndr 13(2019) pp. 616-21. [PubMed: 30641776]

[47]. Nogueira-Machado JA, Chaves MM, From hyperglycemia to AGE-RAGE interaction on the cell surface: a dangerous metabolic route for diabetic patients, Expert Opin Ther Targets12(2008) pp. 871-82. [PubMed: 18554155]

[48]. Ayala A, Munoz MF, Arguelles S, Lipid peroxidation: production, metabolism, and signaling mechanisms of malondialdehyde and 4-hydroxy-2-nonenal, Oxid Med Cell Longev2014(2014) pp. 360438. [PubMed: 24999379]

[49]. Su Z, Li Y, James JC, McDuffie M, Matsumoto AH, Helm GA, et al., Quantitative trait locus analysis of atherosclerosis in an intercross between $\mathrm{C} 57 \mathrm{BL} / 6$ and $\mathrm{C} 3 \mathrm{H}$ mice carrying the mutant apolipoprotein E gene, Genetics172(2006) pp. 1799-807. [PubMed: 16387874]

[50]. Wang SS, Schadt EE, Wang H, Wang X, Ingram-Drake L, Shi W, et al., Identification of pathways for atherosclerosis in mice: integration of quantitative trait locus analysis and global gene expression data, Circ Res101(2007) pp. e11-30. [PubMed: 17641228]

[51]. Li Q, Li Y, Zhang Z, Gilbert TR, Matsumoto AH, Dobrin SE, et al., Quantitative trait locus analysis of carotid atherosclerosis in an intercross between $\mathrm{C} 57 \mathrm{BL} / 6$ and $\mathrm{C} 3 \mathrm{H}$ apolipoprotein $\mathrm{E}$ deficient mice, Stroke39(2008) pp. 166-73. [PubMed: 18048852]

[52]. Watson CT, Kos JT, Gibson WS, Newman L, Deikus G, Busse CE, Smith ML, Jackson KJ5, Collins AM. A comparison of immunoglobulin IGHV, IGHD and IGHJ genes in wild-derived and classical inbred mouse strains. Immunol Cell Biol. 20198 23. In press.

[53]. Cui J, Xiao Y, Shi YH, Wang B, Le GW. Lipoic acid attenuates high-fat-diet-induced oxidative stress and B-cell-related immune depression. Nutrition 28(2012) pp. 275-80. [PubMed: 22305006] 


\section{Highlights}

- $\quad$ Circulating levels of malondialdehyde (MDA), a marker of oxidative stress were found controlled by a significant QTL on chromosome 1 and two suggestive QTLs on chromosomes 1 and 4.

- $\quad$ The QTL Mda1 for MDA coincided precisely with loci for LDL, triglyceride, glucose, and body weight in the confidence interval.

- Plasma LDL, triglyceride, and glucose levels accounted for 25.5, 19.2, and $24.2 \%$ of the variance in circulating MDA levels of F2 mice, respectively.

- $\quad$ After correction for triglyceride or LDL, QTLs for MDA on chromosomes 1 and 4 disappeared. 


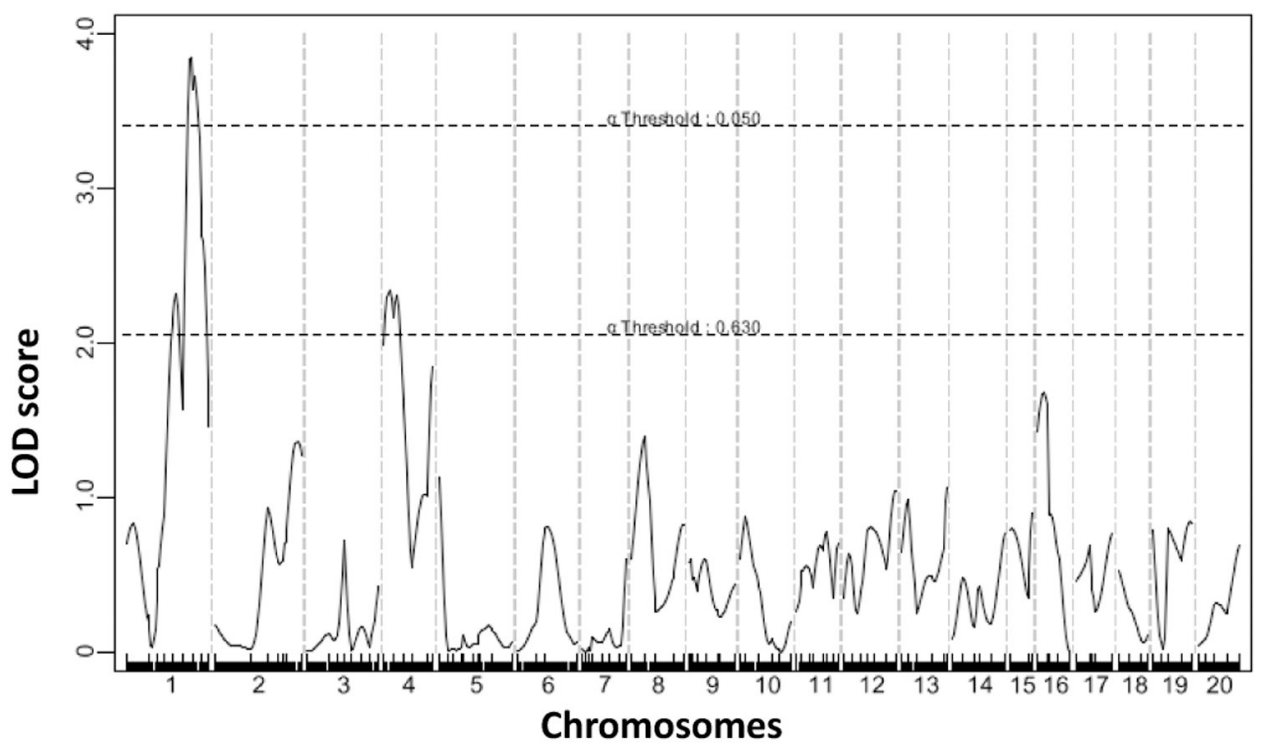

Figure 1.

A genome-wide scan to search for loci affecting plasma MDA levels of female F2 mice derived from B6 and BALB Apoe $\mathrm{e}^{-/-}$mice.

Chromosomes 1-20 are represented numerically on the $\mathrm{X}$-axis. The relative width of the space allotted for each chromosome reflects the relative length of each chromosome. The Yaxis represents the logarithm of odds (LOD) score. Two horizontal dashed lines denote genome-wide thresholds for suggestive $(p=0.63)$ and significant $(p=0.05)$ linkage. 

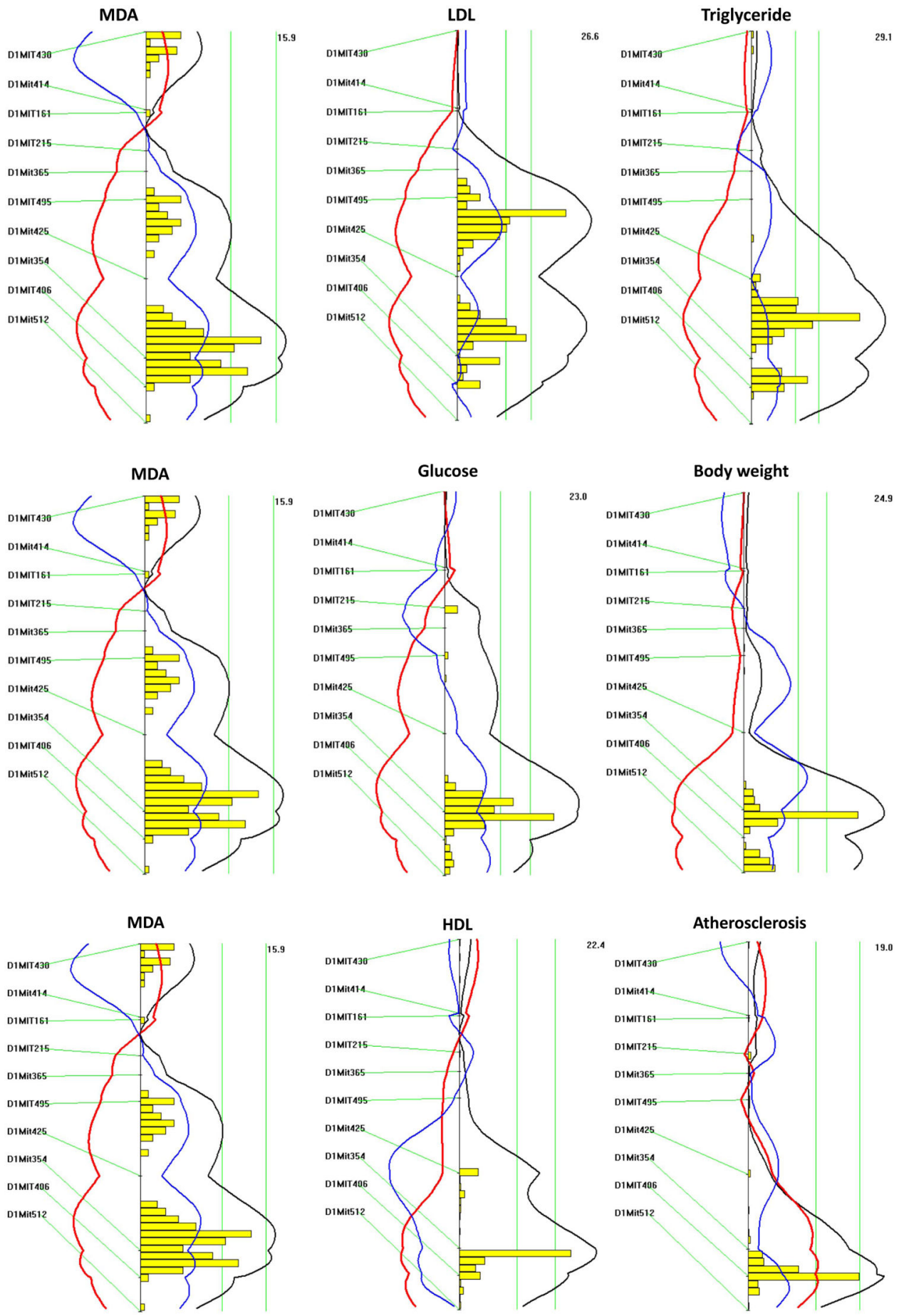

Figure 2.

Interval mapping graphs for plasma MDA, LDL, HDL cholesterol, triglyceride, glucose levels, body weight, and aortic atherosclerosis on chromosome 1 .

Plots were created with the interval mapping function of Map Manager QTX. Yellow histograms estimate the confidence interval of QTL. Two green horizontal lines represent genome-wide significance thresholds for suggestive or significant linkage ( $p=0.63$ and $p=$ 
0.05 , respectively). Black plots reflect the LOD calculated at 1-cM intervals; blue plots, the effect of the high BALB allele; and red plots, the effect of the low B6 allele. 

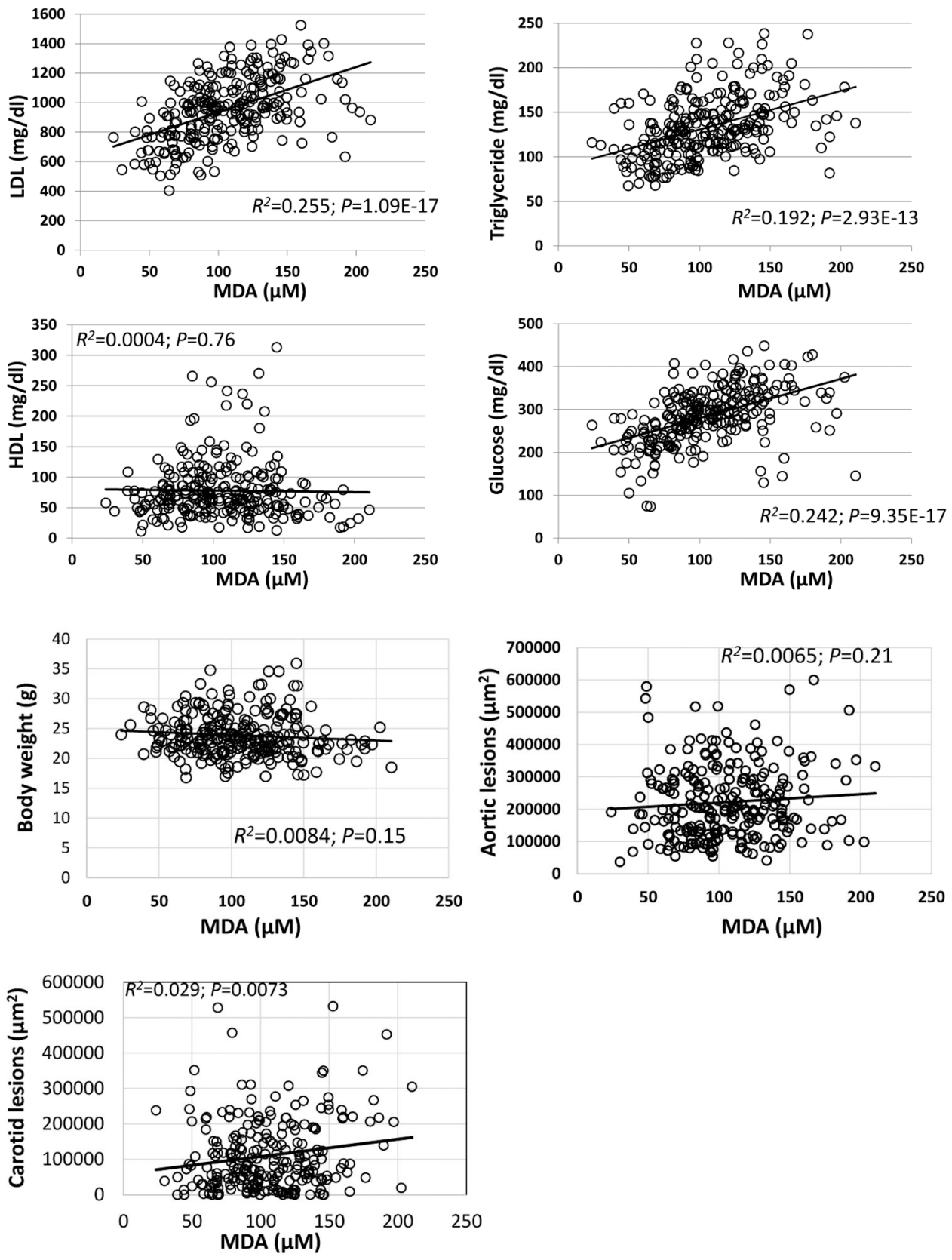

Figure 3.

Regression analysis to evaluate the contribution of plasma LDL, HDL cholesterol, triglyceride, glucose levels, body weight, aortic and carotid atherosclerosis to variation in plasma MDA levels of the F2 mice.

Each point represents values of an individual F2 mouse. The $R^{2}$ and $p$ values are shown in the figures. 

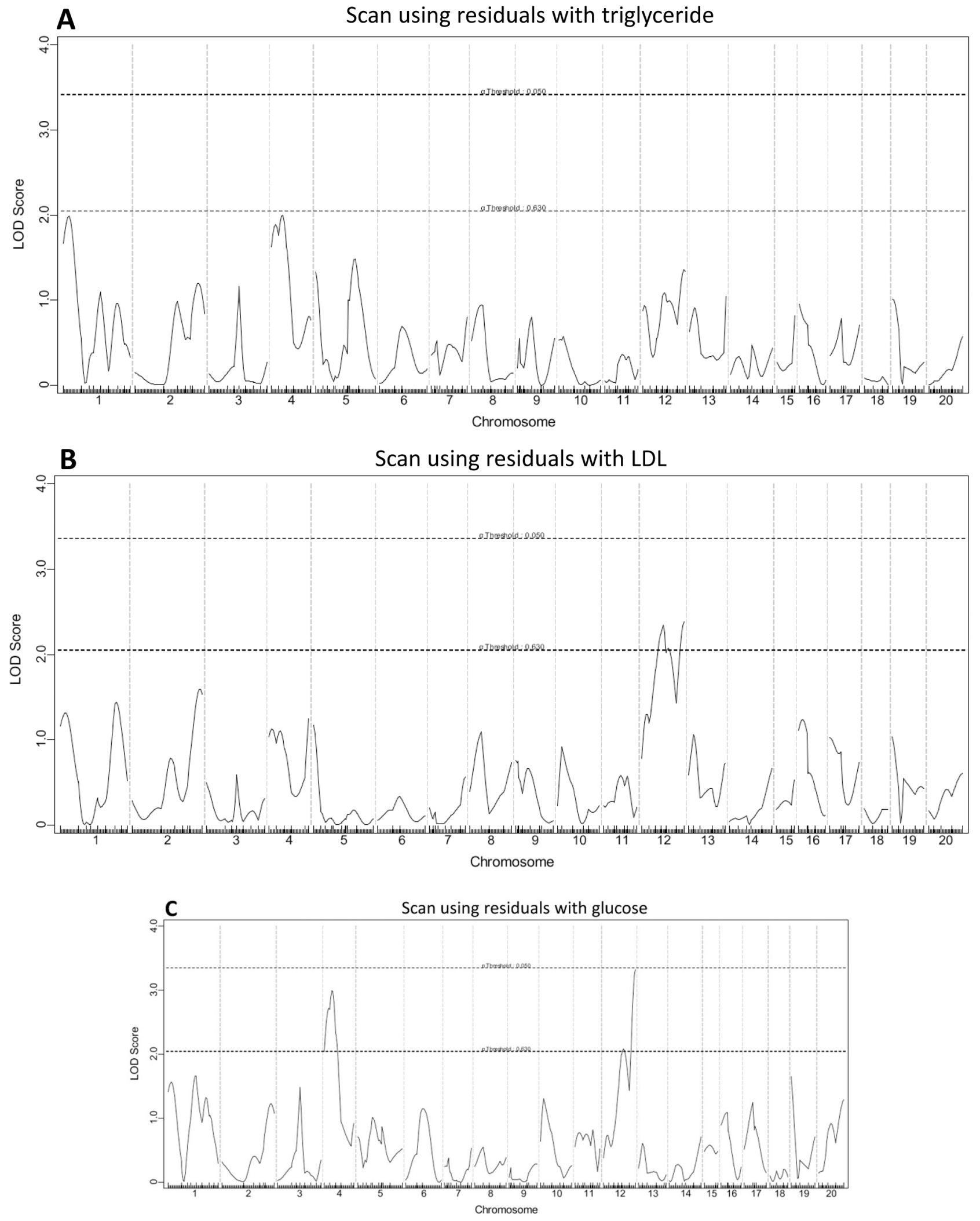

Atherosclerosis. Author manuscript; available in PMC 2021 January 01. 

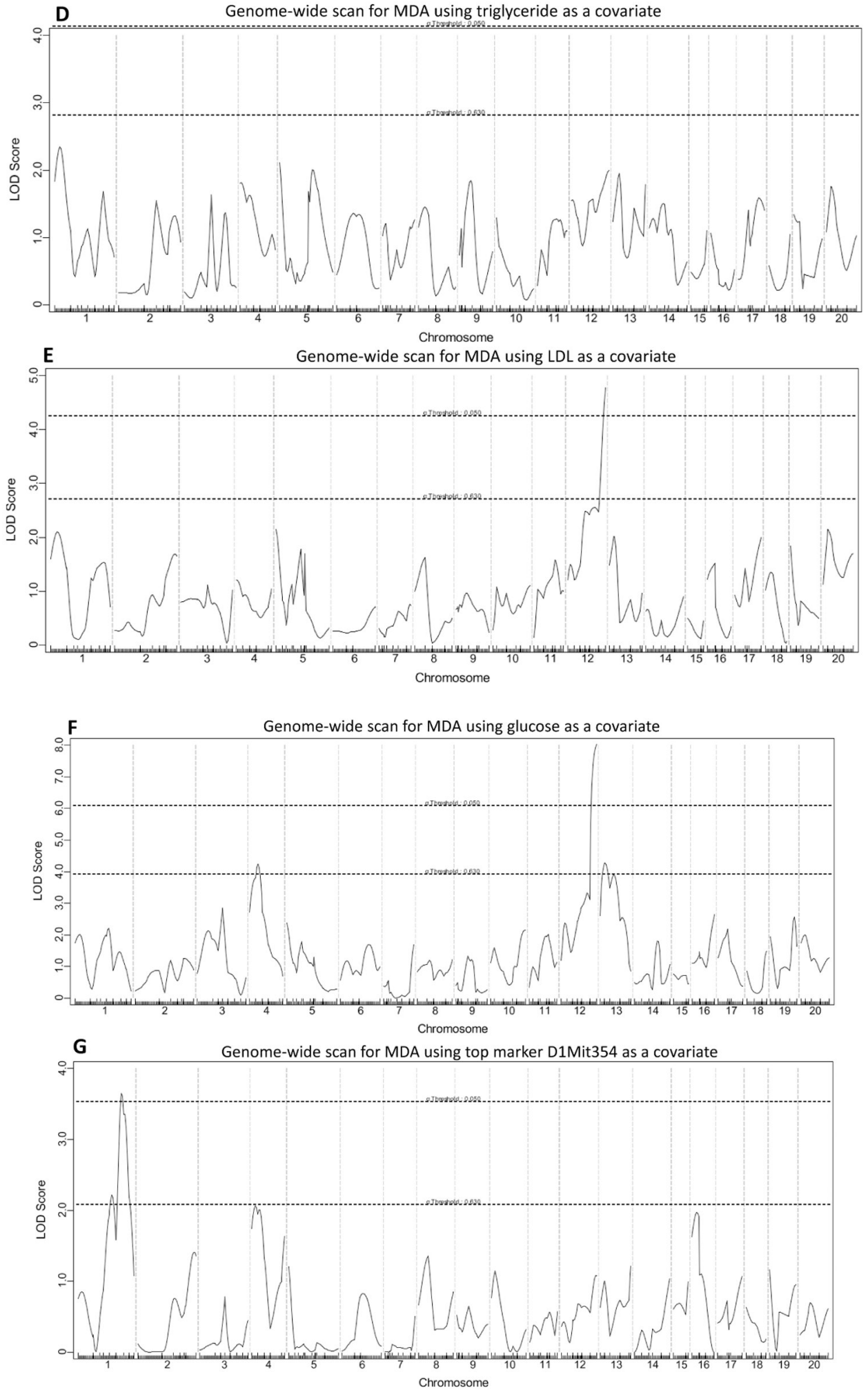

Figure 4.

Genome-wide scans to assess the dependence of QTLs for MDA levels on a particular lipid or glucose trait or a particular genetic marker in F2 mice.

Atherosclerosis. Author manuscript; available in PMC 2021 January 01. 
The residuals derived from the linear regression of MDA levels with triglyceride, LDL or glucose levels were treated as a new phenotype, and the same genome-wide mapping algorithm used in Fig. 1 was performed on the new phenotype to identify MDA QTLs independent of the particular metabolic trait (triglyceride, LDL or glucose levels). Alternatively, the same mapping algorithm used in Fig. 1 was performed except for the use of a lipid or glucose trait as an interactive covariate. (A) A genome-wide scan for detecting QTLs for MDA using the residuals from the linear regression of MDA with triglyceride levels; (B) a genome-wide scan for MDA using residuals from the linear regression of MDA with LDL levels; (C) a genome-wide scan for MDA using residuals from the linear regression of MDA with glucose levels; (D) a genome-wide scan for MDA levels using triglyceride levels as a covariate; (E) a genome-wide scan for MDA levels using LDL levels as a covariate; (F) a genome-wide scan for MDA levels using glucose levels as a covariate; (G) a genome-wide scan for MDA levels using the closest linked marker D1Mit354 to Mda1 as a covariate. 


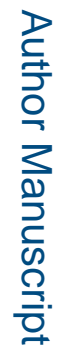

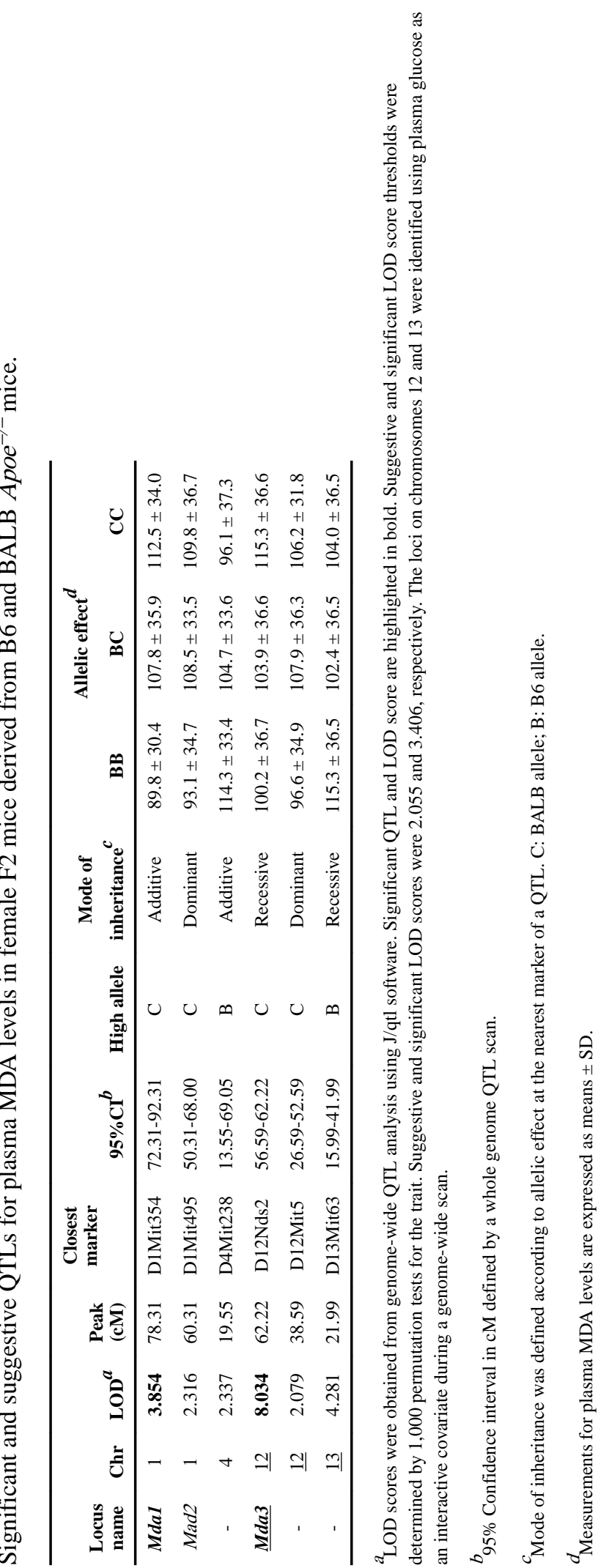

Atherosclerosis. Author manuscript; available in PMC 2021 January 01. 